\title{
Adjuvant Hepatic Arterial Infusion Pump Chemotherapy After Resection of Colorectal Liver Metastases: Results of a Safety and Feasibility Study in The Netherlands
}

Florian E. Buisman, $\mathrm{MD}^{1}$, Dirk J. Grünhagen, $\mathrm{MD}, \mathrm{PhD}^{1}$, Marjolein Y. V. Homs, $\mathrm{MD}, \mathrm{PhD}^{2}$, Cecile Grootscholten, $\mathrm{MD}, \mathrm{PhD}^{3}$, Wills F. Filipe, $\mathrm{MD}^{1}$, Nancy E. Kemeny, $\mathrm{MD}^{4}$, Andrea Cercek, $\mathrm{MD}^{4}$, Micheal I. D'Angelica, $\mathrm{MD}^{5}$, Maarten L. Donswijk, $\mathrm{MD}^{6}$, Leni van Doorn, $\mathrm{NP}^{2}$, Jasper Emmering, MD, $\mathbf{P h D}^{7}$, William R. Jarnagin, $\mathrm{MD}^{5}$, T. Peter Kingham, $\mathrm{MD}^{5}$, Elisabeth G. Klompenhouwer, $\mathrm{MD}$, $\mathbf{P h D}^{8}$, Niels F. M. Kok, MD, PhD ${ }^{9}$, Maria C. Kuiper, NP $^{3}$, Adriaan Moelker, MD, PhD $^{7}$, Warner Prevoo, MD $^{8}$, Michelle W. J. Versleijen, MD, PhD ${ }^{6}$, Cornelis Verhoef, MD, PhD ${ }^{1}$, Koert F. D. Kuhlmann, MD, PhD ${ }^{9}$, and Bas Groot Koerkamp, MD, PhD ${ }^{1}$

${ }^{1}$ Department of Surgery, Erasmus MC Cancer Institute, Erasmus University, Rotterdam, The Netherlands; ${ }^{2}$ Department of Medical Oncology, Erasmus MC Cancer Institute, Erasmus University, Rotterdam, The Netherlands; ${ }^{3}$ Department of Medical Oncology, The Netherlands Cancer Institute, Amsterdam, The Netherlands; ${ }^{4}$ Department of Medical Oncology, Memorial Sloan Kettering Cancer Center, New York, NY; ${ }^{5}$ Department of Surgery, Memorial Sloan Kettering Cancer Center, New York, NY; ${ }^{6}$ Department of Nuclear Medicine, The Netherlands Cancer Institute, Amsterdam, The Netherlands; ${ }^{7}$ Department of Radiology and Nuclear Medicine, Erasmus MC, Erasmus University, Rotterdam, The Netherlands; ${ }^{8}$ Department of Radiology, The Netherlands Cancer Institute, Amsterdam, The Netherlands; ${ }^{9}$ Department of Surgery, The Netherlands Cancer Institute, Amsterdam, The Netherlands

\begin{abstract}
Background. The 10-year overall survival with adjuvant hepatic arterial infusion pump (HAIP) chemotherapy after resection of colorectal liver metastases (CRLMs) was 61\% in clinical trials from Memorial Sloan Kettering Cancer Center. A pilot study was performed to evaluate the safety and feasibility of adjuvant HAIP chemotherapy in patients with resectable CRLMs.

Study Design. A phase II study was performed in two centers in The Netherlands. Patients with resectable CRLM without extrahepatic disease were eligible. All patients underwent complete resection and/or ablation of CRLMs and pump implantation. Safety was determined by the 90-day HAIP-related postoperative complications from the day of pump placement (Clavien-Dindo classification,
\end{abstract}

(C) The Author(s) 2019

First Received: 12 August 2019; Published Online: 22 October 2019

B. Groot Koerkamp, MD, PhD

e-mail: b.grootkoerkamp@erasmusmc.nl grade III or higher) and feasibility by the successful administration of the first cycle of HAIP chemotherapy.

Results. A total of 20 patients, with a median age of 57 years (interquartile range [IQR] 51-64) were included. Grade III or higher HAIP-related postoperative complications were found in two patients (10\%), both of whom had a reoperation (without laparotomy) to replace a pump with a slow flow rate or to reposition a flipped pump. No arterial bleeding, arterial dissection, arterial thrombosis, extrahepatic perfusion, pump pocket hematoma, or pump pocket infections were found within 90 days after surgery. After a median of 43 days (IQR 29-52) following surgery, all patients received the first dose of HAIP chemotherapy, which was completed uneventfully in all patients.

Conclusion. Pump implantation is safe, and administration of HAIP chemotherapy is feasible, in patients with resectable CRLMs, after training of a dedicated multidisciplinary team. 
Recurrent disease is reported in up to $70 \%$ of patients after resection of colorectal liver metastases (CRLMs). ${ }^{1}$ Reported 5- and 10-year overall survival (OS) of CRLM patients treated with resection and systemic chemotherapy were $40 \%$ and $25 \%$, respectively. ${ }^{1}$

The rationale of adjuvant hepatic arterial infusion pump (HAIP) chemotherapy after resection of CRLM is that initial recurrences involve the liver in half of the patients. HAIP chemotherapy involves a subcutaneous surgically implanted pump that delivers chemotherapy through a catheter directly into the hepatic artery via the gastroduodenal artery (GDA). Arterial administration is preferred because liver tumors mainly depend on arterial rather than portal venous blood supply. ${ }^{2,3}$ Floxuridine (or FUDR) is the preferred drug for HAIP chemotherapy. Due to its high hepatic extraction rate, intratumoral exposure is up to 400-fold higher compared with systemic administration, with little or no systemic toxicity. ${ }^{4}$ Two randomized controlled trials (RCTs) performed in the 1990s demonstrated superior OS of HAIP chemotherapy. Moreover, the 10-year OS of patients who received adjuvant HAIP chemotherapy in several phase II trials after 2003 was $61 \%$. $^{5,6}$

Regardless of these impressive results, HAIP chemotherapy is not commonly used outside of Memorial Sloan Kettering Cancer Center (MSKCC). One of the barriers is that floxuridine is not registered in the European Union (EU). Moreover, HAIP chemotherapy requires comprehensive training of and commitment from a multidisciplinary team.

Previous studies demonstrated both safety and feasibility concerns due to its complexity, requiring both technical knowledge and practical skills..$^{7-9}$ However, a previous study of 544 patients demonstrated that an experienced team was associated with less pump-related complications. The pump failure rate was only 5\% in the first 6 months after implantation. ${ }^{2}$ HAIP-related postoperative complications include pump flow-rate abnormalities, pump dislocation, arterial bleeding, arterial dissection, arterial thrombosis, extrahepatic perfusion, pump pocket hematoma, and pump pocket infections. ${ }^{8}$

The aim of this study was to determine the safety and feasibility of adjuvant HAIP chemotherapy after resection of CRLMs in two centers in The Netherlands.

\section{METHODS}

\section{Study Design}

A phase II, multicenter, single-arm, safety and feasibility study was conducted from February 2018 to February 2019 at the Erasmus MC Cancer Institute (Rotterdam) and The Netherlands Cancer Institute (Amsterdam) in The
Netherlands. The Institutional Review Board approved the study protocol (MEC-2017-282). The study was registered in the Netherlands Trial Register (number 6917).

\section{Patients}

All patients with histologically confirmed colorectal cancer and resectable CRLM without extrahepatic disease (EHD) were evaluated for inclusion. EHD was defined as any disease outside the liver prior to or at time of diagnosis of CRLM. Patients with EHD found at surgery were excluded. Patients were also excluded if positioning of a catheter for HAIP chemotherapy was not feasible based on a preoperative arterial computed tomography (CT) scan, prior hepatic radiation or resection, CRLM requiring twostaged resection, liver-first approach, and diagnosis of another malignancy. Positioning of a catheter was not considered feasible if the GDA had no connecting branch to the left or right liver (e.g. in a patient with a completely replaced right and left hepatic artery).

\section{Training}

The initial eight implantations (four in each center) were performed under the supervision of surgeons from MSKCC (MD and TK), who both have over 10-years of experience in pump implantations (i.e. a total of more than 200 implantations each). The multidisciplinary teams of both participating centers visited MSKCC for a 2-day workshop. Additional training involved detailed protocols and video material of the surgical pump implantation. A PhD student attended all implantations, supervised pump refills, and provided hands-on workshops for nurses and staff members.

\section{Surgical Procedure}

A dedicated team of two surgeons in each center performed all implantations. Surgical resection of CRLMs by laparotomy, with or without resection of the primary tumor, was combined with implantation of the HAI pump with a constant non-programmable flow rate (Tricumed IP2000 V). This pump has similar specifications as the pump used in MSKCC (Codman 3000), with the main difference being that the reservoir of the Tricumed pump is pressurized by butane rather than Freon, which is not allowed in the EU due to environmental laws. Treatment of the CRLMs involved complete resection and/or open ablation. In case of a simultaneous resection of the primary tumor, liver resection with pump implantation was performed first, followed by resection of the primary tumor to prevent contamination of the pump. Prior to pump implantation, a function test was performed to check the 
adequate operation of the pump. A cholecystectomy was performed to avoid cholecystitis as a result of intra-arterial chemotherapy through the cystic artery. ${ }^{10}$ The pump pocket was created at the left-lower quadrant of the abdominal wall, or in the right-lower quadrant in patients with a colostomy. The pocket cavity was created threequarters caudal to the incision to ensure easy access of the pump septum for percutaneous refills.

The entire GDA, as well as the proximal proper hepatic artery, were mobilized and dissected circumferentially from their attachments to facilitate insertion of the catheter and to avoid inadvertent perfusion of the pancreas, stomach, or duodenum. The distal GDA was ligated with a nonabsorbable tie and a transversal arteriotomy was performed followed by insertion of the catheter. The catheter was positioned just at the origin of the GDA. Positioning of the catheter with the tip in the hepatic artery may cause turbulence and the risk of thrombosis, while positioning the catheter too far from the hepatic artery may cause pooling of floxuridine in the GDA, with a risk of erosion, a pseudoaneurysm, and hemorrhage. A metal connector was used to connect a commercially available (B. Braun Celsite ${ }^{\circledR}$ ) intra-arterial catheter (distal catheter) with the Tricumed catheter that comes with the pump (Tricumed Catheter $\left.1000^{\circledR}\right)$. This connection was secured with two non-absorbable ties. The distal catheter has several beads (i.e. local thickening of the catheter wall), which were used to secure the catheter with non-absorbable ties in the GDA. Perfusion of both lobes of the liver and lack of extrahepatic perfusion was confirmed by an intraoperative bolus injection of methylene blue. After the perfusion test, the catheter was flushed with heparinized saline, and the wounds were closed. Any replaced and accessory hepatic arteries were ligated, provided that a patent GDA connected with at least one hepatic artery was present. Intrahepatic shunts will typically reassure that the catheter perfuses all liver segments, which was confirmed intraoperatively, and during follow-up with postoperative scintigraphy.

\section{Postoperative Procedures}

Prior to the start of HAIP chemotherapy, a postoperative technetium-99-labeled macroaggregated albumin (Tc-99m MAA) scintigraphy was performed to again confirm the absence of extrahepatic perfusion. In case of extrahepatic perfusion, patients were evaluated angiographically and branches were embolized with re-testing prior to the start of treatment.

\section{Chemotherapeutic Regimen}

HAIP chemotherapy was initiated 4-12 weeks after surgery depending on the patients' condition and liver function. The pump was refilled with a heparinized saline solution (35,000 IE in $35 \mathrm{~mL} \mathrm{NaCl} 0.9 \%$ ) every 2 weeks until the start of HAIP chemotherapy to prevent thrombosis of the catheter. All patients were scheduled for six cycles of 4 weeks of HAIP chemotherapy with floxuridine. Each cycle comprised 2 weeks of HAIP chemotherapy followed by a 2-week rest period during which the pump was filled with the heparinized saline solution. Floxuridine was administered based on the MSKCC regimen $(0.12 \mathrm{mg} /$ $\mathrm{kg} /$ day). ${ }^{11,12}$ If the actual weight was more than $25 \%$ above the ideal weight, the dose of floxuridine was calculated using the average of the actual and ideal weight. Floxuridine was administered in a solution of 35,000 IE heparin and $25 \mathrm{mg}$ dexamethasone in $\mathrm{NaCl} 0.9 \%$ with a total volume of $35 \mathrm{ml}$. A prophylactic dose of $20 \mathrm{mg}$ of proton pump inhibitors was administered daily during HAIP chemotherapy. No adjuvant systemic chemotherapy was administered since this is not the standard of care in The Netherlands.

\section{Outcomes}

Safety was determined by the percentage of postoperative complications (Clavien-Dindo classification grade III or higher) within 90 days after surgery related to HAI pump placement. The feasibility was defined as the percentage of patients receiving at least one cycle of adjuvant HAIP chemotherapy after resection of CRLMs.

\section{Definitions and Statistical Analysis}

Demographic and clinicopathological characteristics were presented as medians with interquartile ranges (IQRs), and as means with ranges for continuous variables and proportions for categorical variables. CRLMs that were detected within 3 months of resection of the primary tumor were considered synchronous. Any chemotherapy administered within 3 months prior to resection was considered as preoperative chemotherapy. A positive resection margin (R1) was defined as tumor cells present at the resection margin, and major liver resection was defined as complete resection of three or more segments. All analyses were performed using SPSS version 24 (IBM Corporation, Armonk, NY, USA).

\section{RESULTS}

A total of 22 patients were included in two centers (Erasmus MC Cancer Institute and The Netherlands Cancer Institute, The Netherlands) from February 2018 until February 2019. Two patients were excluded during surgery; one patient had unresectable CRLMs found during 
intraoperative ultrasonography, and one patient was excluded due to an occult peritoneal lesion that was found during surgery and confirmed by frozen section biopsy. No patients were excluded due to unexpected abnormal hepatic artery anatomy. A total of 20 patients were eligible.

\section{Baseline Characteristics}

Baseline characteristics of 20 patients who were eligible for surgical treatment and pump implantation are shown in Table 1. Median age was 57 years (IQR 51-64), and the majority of patients were male $(n=12,60 \%)$. Most patients had left-sided colorectal cancer $(n=11,55 \%)$, followed by rectal $(n=6,30 \%)$ and right-sided colorectal cancer $(n=3,15 \%)$. About half of the patients had synchronous CRLMs $(n=11,55 \%)$.

\section{Surgical Aspects}

Surgical aspects are summarized in Table 2. Preoperative chemotherapy was administered in seven patients (35\%). In four patients (20\%), the procedure was combined with simultaneous resection of the primary tumor; in seven patients (35\%), ablation was combined with resection; and in two patients $(10 \%)$, only open ablation and pump implantation were performed. A major liver resection was performed in four patients $(20 \%)$. The hepatic arterial anatomy was abnormal in 10 patients $(50 \%)$, requiring ligation of accessory or replaced left and/or right hepatic arteries. The pump was positioned in the left-lower quadrant of the abdomen in 18 patients $(90 \%)$. The right-lower abdomen was the preferred site in two patients $(10 \%)$ due to a prior colostomy in the left-lower quadrant. The median hospital stay was 8 days (IQR 6-9). Postoperative Tch99m MAA scintigraphy showed no signs of extrahepatic perfusion in all patients.

\section{Postoperative Complications}

Postoperative complications are summarized in Table 3. No postoperative 90-day mortality was found. No arterial bleeding, arterial dissection, arterial thrombosis, pump pocket hematoma, or pump pocket infections were found within the first 90 days after surgery. Five patients $(25 \%)$ had postoperative complications of grade III or higher. Two patients $(10 \%)$ had complications related to HAI pump placement; the first patient required pump replacement due to a decreased flow rate of the pump, and the second patient had a flipped pump (upside down) requiring reoperation. In both patients, reoperation involved a local exploration of the pump pocket without a laparotomy, with same-day discharge. Both patients recovered uneventful and continued HAIP chemotherapy within 2 weeks.
Another three patients (15\%) required re-interventions due to complications unrelated to HAI pump implantation. One patient required a re-laparotomy for biliary peritonitis as a result of biliary leakage at the liver resection margin, as well as a second re-laparotomy due to fascial dehiscence. A second patient was readmitted with an intraabdominal fluid collection that was treated with both percutaneous drainage and intravenous antibiotics. The third patient was readmitted for percutaneous drainage of an intra-abdominal fluid collection with negative culture. All three patients recovered uneventful.

\section{Initiation of Hepatic Arterial Infusion Pump (HAIP) Chemotherapy}

The median period to administration of the first cycle of HAIP chemotherapy was 43 days (IQR 29-52). Percutaneous access of the pump for the first cycle of HAIP chemotherapy was performed without adverse events in all patients. All patients uneventfully completed the first cycle of HAIP chemotherapy, which was the primary endpoint for feasibility.

\section{DISCUSSION}

This study demonstrated that HAI pump implantation and administration of adjuvant HAIP chemotherapy in patients with resectable CRLMs is safe and feasible in The Netherlands. Safety was demonstrated as two patients (10\%) developed HAIP-related postoperative complications that were resolved with a reoperation to replace or reposition the subcutaneous pump. Feasibility was demonstrated because all patients started HAIP chemotherapy within 6 weeks after surgery.

As a result of the pump with a decreased flow rate, the pre-implantation pump performance test procedure was adapted. The pump flow rate is temperature-dependent, reaching optimal flow rates at body temperature. The new pump performance test included continuous heating of the pump to $37{ }^{\circ} \mathrm{C}$ within an ex vivo heater, allowing precise observation of pump flow rate mimicking in vivo conditions. In order to minimize the risk of pump dislocation (flipping within the pump pocket), all pockets were created with minimal residual space in order to achieve a tight fit with minimal risk of dislocation of the infusion pump in the pocket. In obese patients (i.e. BMI $>30$ ) we prefer to position the pump on the chest wall. The observed complication rate seems acceptable compared with a large retrospective study in which 544 patients who underwent pump implantation for CRLMs were evaluated. ${ }^{8}$ Pumprelated complications were reported in 120 patients $(22 \%)$ and were classified as related to the hepatic arterial system 
TABLE 1 Baseline characteristics

\begin{tabular}{|c|c|c|c|c|c|c|c|c|c|c|c|c|}
\hline $\begin{array}{l}\text { Median } \\
\text { (IQR) or } \\
n(\%)\end{array}$ & $\begin{array}{l}\text { Age } \\
\text { (years) } \\
57 \\
(51-64)\end{array}$ & $\begin{array}{l}\text { Male: } \\
12(60)\end{array}$ & $\begin{array}{l}\text { ASA } \\
\text { score } \\
2 \\
(1-2)\end{array}$ & $\begin{array}{l}\text { BMI } \\
\left(\mathrm{kg} / \mathrm{m}^{2}\right) \\
27 \\
(24-27)\end{array}$ & $\begin{array}{l}\text { Location } \\
\text { CRC } \\
\text { Right: } 3 \\
(15)\end{array}$ & $\begin{array}{l}\mathrm{T}- \\
\text { stage } \\
\text { CRC } \\
3 \\
(3-4)\end{array}$ & $\begin{array}{l}\text { Nodal } \\
\text { status } \\
\text { N0: } 6 \\
(30)\end{array}$ & $\begin{array}{l}\text { Synchronous/ } \\
\text { metachronous } \\
\text { Syn: } 11 \text { (55) }\end{array}$ & $\begin{array}{l}\begin{array}{l}\text { DFI } \\
\text { (months) }\end{array} \\
1(0-13)\end{array}$ & $\begin{array}{l}\text { No. of } \\
\text { CRLMs } \\
2(1-5)\end{array}$ & $\begin{array}{l}\text { Size of the } \\
\text { largest } \\
\text { CRLM }(\mathrm{cm}) \\
2.3(0.8-7.1)\end{array}$ & $\begin{array}{l}\text { CEA } \\
(\mu \mathrm{g} / \mathrm{L}) \\
6 \\
(3-26)\end{array}$ \\
\hline Case 1 & 58 & Female & 3 & 27 & Rectum & 3 & 0 & Metachronous & 6 & 1 & 6.7 & 23 \\
\hline Case 2 & 64 & Male & 2 & 34 & Left & 3 & 2 & Metachronous & 41 & 3 & 2.4 & 6 \\
\hline Case 3 & 52 & Female & 2 & 29 & Left & 3 & 1 & Metachronous & 13 & 1 & 2.8 & 5 \\
\hline Case 4 & 64 & Male & 3 & 24 & Left & 3 & 1 & Metachronous & 13 & 1 & 1.8 & 5 \\
\hline Case 5 & 75 & Female & 2 & 26 & Left & 3 & 0 & Synchronous & 0 & 1 & 2.2 & 8 \\
\hline Case 6 & 67 & Male & 1 & 24 & Left & 4 & 0 & Synchronous & 0 & 2 & 4.8 & 34 \\
\hline Case 7 & 50 & Female & 1 & 24 & Left & 3 & 0 & Synchronous & 2 & 2 & 2.0 & 63 \\
\hline Case 8 & 54 & Male & 3 & 24 & Right & 4 & 2 & Metachronous & 28 & 2 & 2.3 & 27 \\
\hline Case 9 & 68 & Male & 2 & 24 & Right & 4 & 2 & Synchronous & 0 & 5 & 1.2 & 40 \\
\hline Case 10 & 58 & Male & 2 & 25 & Left & 3 & 1 & Synchronous & 0 & 5 & 7.1 & 2 \\
\hline Case 11 & 57 & Female & 2 & 25 & Left & 4 & 2 & Metachronous & 15 & 2 & 4.8 & 1 \\
\hline Case 12 & 66 & Male & 3 & 23 & Left & 4 & 1 & Synchronous & 0 & 4 & 4.2 & 24 \\
\hline Case 13 & 51 & Female & 1 & 27 & Rectum & 3 & 1 & Metachronous & 2 & 5 & 1.3 & 3. \\
\hline Case 14 & 42 & Female & 1 & 23 & Left & 3 & 0 & Metachronous & 4 & 13 & 1.5 & 3 \\
\hline Case 15 & 54 & Female & 1 & 25 & Right & 3 & 1 & Synchronous & 0 & 3 & 2.5 & 19 \\
\hline Case 16 & 61 & Male & 2 & 25 & Left & 3 & 1 & Synchronous & 0 & 2 & 1.6 & 3 \\
\hline Case 17 & 54 & Male & 2 & 28 & Rectum & 3 & 1 & Synchronous & 0 & 7 & 52 & 880 \\
\hline Case 18 & 43 & Male & 1 & 25 & Rectum & 2 & 1 & Synchronous & 0 & 12 & 0.8 & 4 \\
\hline Case 19 & 57 & Male & 1 & 24 & Rectum & 3 & 0 & Metachronous & 12 & 2 & 2.2 & 6 \\
\hline Case 20 & 46 & Male & 2 & 24 & Rectum & 2 & 1 & Synchronous & 0 & 1 & 1.0 & 3 \\
\hline
\end{tabular}

ASA American Society of Anesthesiologists, BMI body mass index, $C E A$ carcinoembryonic antigen, $C R C$ colorectal cancer, $C R L M$ colorectal liver metastasis, DFI disease-free interval, IQR interquartile range, $N O$ node-negative, Syn synchronous, T-stage CRC tumor-stage colorectal cancer

$(n=62,51 \%)$, the catheter $(n=33,26 \%)$, the pumppocket $(n=19,16 \%)$, or the pump $(n=6,5 \%)$. Technical complications could be salvaged in 54 patients $(45 \%)$. A higher rate of complications was found with surgeons who performed less than 25 implantations (31\% vs. 19\%; $p<0.001)$. Other perioperative factors were comparable with our study: mean operative time (260 $\mathrm{min}$ vs. $241 \mathrm{~min}$ in our study), mean blood loss ( $490 \mathrm{~mL}$ vs. $724 \mathrm{~mL}$ in our study), and length of hospital stay ( 8 days vs. 9 days in our study). However, long-term follow-up is needed for complete comparison of our results with this study.

Our multidisciplinary approach with extensive training and proctoring by MSKCC was essential for the safety and feasibility of setting up an HAIP chemotherapy program. In a previous RCT on hepatic arterial infusion (HAI) chemotherapy, lack of training and experience appeared to be the major factor for the failure of safety and feasibility. ${ }^{7}$ Lorenz et al. compared resection of CRLMs combined with adjuvant HAI of 5-fluorouracil, with resection of CRLM alone. The trial was prematurely terminated after interim analysis for futility. At the time of interim analysis, a total of 113 patients were randomized into each group. Eight patients $(7 \%)$ within the HAI group died within 30 days after surgery; four deaths were related to HAI chemotherapy toxicity, three were related to catheter-related bleeding, and one was related to to angiography-induced shock. In the control group, three patients (3\%) died within 30 days. High rates of dropouts, i.e. patients who did not receive the assigned treatment, were reported in both groups, with various reasons: 24 patients $(21 \%)$ assigned to HAI + resection (no catheter implanted [ $n=7]$, CRLM not resected $[n=6]$, malperfusion $[n=5]$, refusal of patient $[n=2]$, port complications $[n=2]$, liver cirrhosis $[n=1]$, and postoperative ileus $[n=1]$ ), and 13 patients $(12 \%)$ assigned to resection alone (CRLM not resected $[n=10]$ and residual disease after resection $[n=3]$ ). Explanations that could have accounted for the failure of this trial included participation of 26 centers, with each center performing only approximately one intra-arterial catheter placement per year; the use of a port with a catheter in the flow of the 


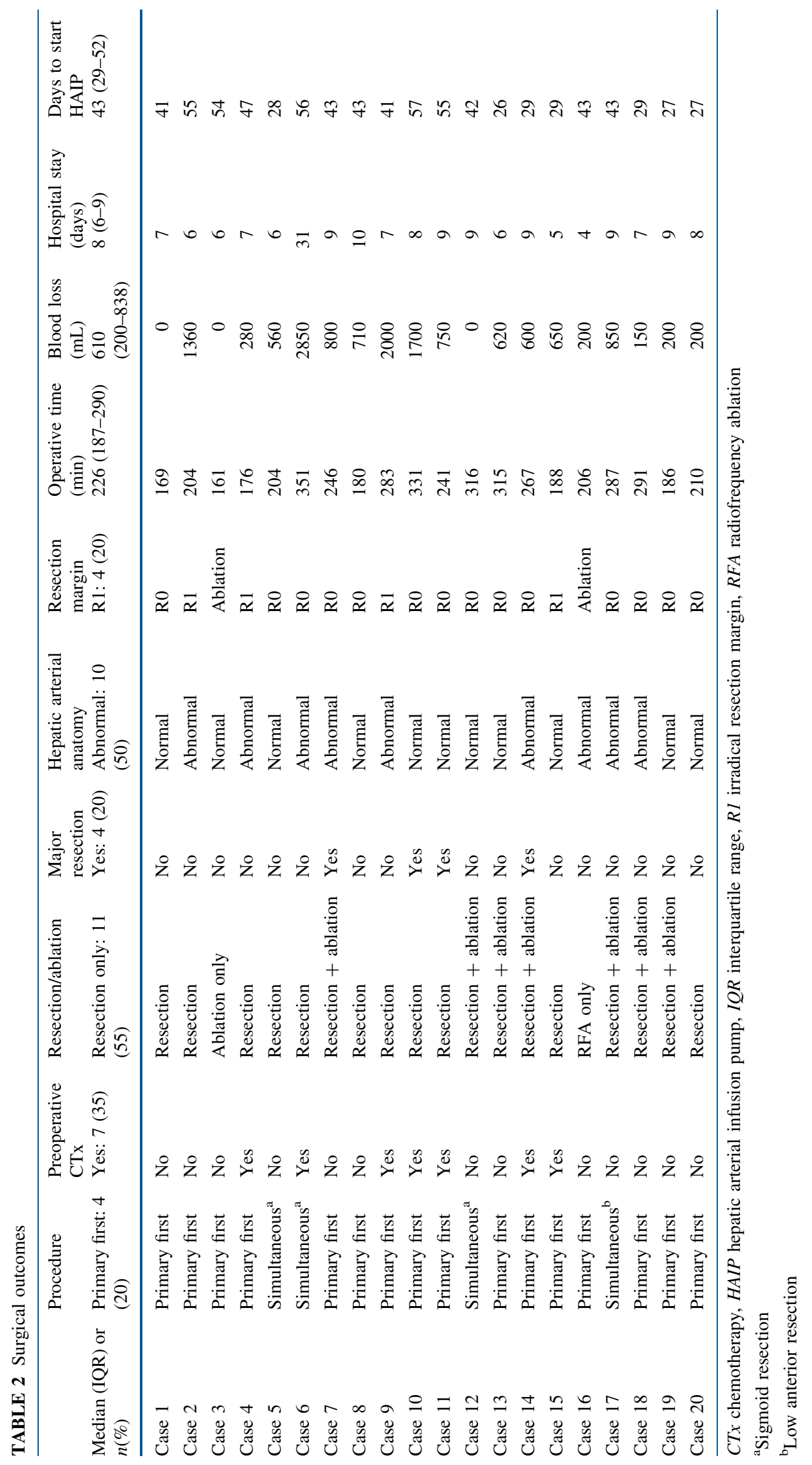


TABLE 3 Postoperative complications within 90 days of surgery

\begin{tabular}{|c|c|c|c|c|c|c|}
\hline $\begin{array}{l}\text { Total } \\
(\%)\end{array}$ & $\begin{array}{l}\text { Grade III or higher } \\
\text { (Clavien-Dindo) } \\
5(25)\end{array}$ & $\begin{array}{l}\text { HAI } \\
\text { pump- } \\
\text { related } \\
2(10)\end{array}$ & $\begin{array}{l}\text { Time to } \\
\text { event (days) }\end{array}$ & $\begin{array}{l}\text { Requiring } \\
\text { readmission } \\
4(20)\end{array}$ & $\begin{array}{l}\text { Requiring } \\
\text { surgery }\end{array}$ & Specified \\
\hline Case 1 & - & & & & & \\
\hline Case 2 & $\mathrm{IIIb}$ & Yes & 42 & Yes & Yes & Pump replacement due to slow flow rate \\
\hline Case 3 & $\mathrm{IIIb}$ & Yes & 41 & Yes & Yes & Flipped pump \\
\hline Case 4 & - & & & & & \\
\hline Case 5 & - & & & & & \\
\hline Case 6 & IVa & No & 4 & No & Yes $(2 \times)$ & $\begin{array}{l}\text { 1. Biliary peritonitis due to biliary leakage at the } \\
\text { liver resection margin } \\
\text { 2. Threatening abdominal fascial dehiscence }\end{array}$ \\
\hline Case 7 & - & & & & & \\
\hline Case 8 & - & & & & & \\
\hline Case 9 & - & & & & & \\
\hline Case 10 & IIIa & No & 13 & Yes & No & $\begin{array}{l}\text { Percutaneous drainage of sterile abdominal fluid } \\
\text { collection }\end{array}$ \\
\hline Case 11 & IIIa & No & 9 & Yes & No & $\begin{array}{l}\text { Spontaneous bacterial peritonitis requiring } \\
\text { antibiotics and percutaneous drainage }\end{array}$ \\
\hline Case 12 & - & & & & & \\
\hline Case 13 & - & & & & & \\
\hline Case 14 & - & & & & & \\
\hline Case 15 & - & & & & & \\
\hline Case 16 & - & & & & & \\
\hline Case 17 & - & & & & & \\
\hline Case 18 & - & & & & & \\
\hline Case 19 & - & & & & & \\
\hline Case 20 & - & & & & & \\
\hline
\end{tabular}

HAI hepatic arterial infusion

hepatic artery, resulting in a high rate of technical failures (e.g. hepatic arterial thrombosis); and the use of intra-arterial 5-fluorouracil, which is not only less effective (lower dose due to a smaller first-pass effect) but also has a much higher systemic exposure compared with floxuridine. ${ }^{4}$

Several studies, including an RCT, demonstrated superior survival of HAIP chemotherapy compared with systemic chemotherapy alone in patients with resectable CRLM. ${ }^{5,6,13,14}$ A phase III RCT for adjuvant HAIP chemotherapy found an improvement in 2-year survival ( $86 \%$ vs. $72 \% ; p=0.03) .{ }^{13}$ Long-term follow-up of 287 patients receiving adjuvant HAIP chemotherapy in four prospective trials at MSKCC demonstrated a 10-year OS of $61 \% .^{5}$ A recent propensity scored analysis demonstrated an OS benefit of 23 months of adjuvant HAIP chemotherapy compared with systemic chemotherapy alone (67 months vs. 44 months; $p<0.001) .{ }^{14}$ The OS without HAIP was similar with other large cohorts outside
MSKCC. ${ }^{15}$ The difference remained at propensity score analysis, with an adjusted hazard ratio of 0.67 (95\% confidence interval 0.59-0.76; $p<0.001$ ).

Implementation of HAIP chemotherapy beyond MSKCC is currently limited to a few centers around the world (e.g. University Hospital Zurich, Zurich, Switzerland; University of Pittsburgh Medical Center, Pittsburgh, PA, USA; and Washington University School of Medicine, St. Louis, MO, USA). Several explanations have been suggested that could account for this. The historical perspective may be partly responsible. The first trials on HAIP chemotherapy date from the 1990s, a decade in which new promising agents for systemic chemotherapy such as irinotecan and oxaliplatin were introduced. Administration of intravenous drugs was simple compared with the implementation of HAIP chemotherapy, which required new skills and close collaboration within multidisciplinary teams. Modern systemic chemotherapy results in superior survival in selected patients with stage IV CRC. ${ }^{16,17}$ In the 
subgroup of patients with resectable CRLM, no OS benefit was found $(p=0.30)$ in a phase III RCT, although progression-free survival was superior in the per protocol analysis $(p=0.035) .{ }^{18}$ Despite these results, the recurrence rate was still about $70 \%$. This disappointing high percentage of recurrent disease after curative resection of CRLMs and perioperative systemic chemotherapy has renewed interest in adjuvant HAIP chemotherapy.

Second, regulatory factors have also opposed implementation of HAIP chemotherapy outside the US. Floxuridine was first registered by the US FDA in 1971; however, in the EU, floxuridine cannot be used outside clinical trials since it is not registered. Others have resorted to 5-fluorouracil or oxaliplatin instead of floxuridine. A previous trial on intermittent infusion of 5-fluorouracil through a mediport in the hepatic artery was terminated prematurely, mainly due to a high rate of 5-fluorouracilrelated complications. ${ }^{7}$ However, the hallmark of HAIP chemotherapy is the $95 \%$ first-pass effect of floxuridine in the liver that allows for a very high dosage with continuous infusion without systemic toxicity.

No infusion pump with the intended use of intra-arterial chemotherapy is currently registered in the EU. The Tricumed IP2000 V infusion pump is CE marked and has been used for many years in patients with spasticity and chronic pain. Both registration of floxuridine, and an infusion pump for HAIP chemotherapy, are essential steps for implementation of this treatment in the EU. The subcutaneous pump is a key component of the intra-arterial chemotherapy because floxuridine has a half-life of only $10 \mathrm{~min}^{4}$ A percutaneous approach for the delivery of intraarterial chemotherapy has been investigated by the Gustave Roussy Hospital in Paris (France). ${ }^{19}$ Goéré et al. administered intra-arterial oxaliplatin using a percutaneous catheter in the hepatic artery. With the percutaneous approach, a catheter remains positioned in the flow of the hepatic artery, with a higher risk of hepatic artery thrombosis. Therefore, the percutaneous approach is not suitable for prolonged administration. The pump has an intra-arterial catheter in the GDA, outside the hepatic arterial flow and therefore less likely to cause thrombosis. The pump can stay in for many years for the treatment of disease recurrence in the liver. Furthermore, the surgical approach allows complete circumferential dissection of the artery, which is important to avoid complications of extrahepatic perfusion of floxuridine. The potential effects of extrahepatic perfusion of floxuridine are more severe than the effects of extrahepatic perfusion of oxaliplatin due to the high dose of floxuridine administered compared with oxaliplatin, provided by its high first-pass effect.

\section{CONCLUSIONS}

This is the first study prospectively reporting early safety and feasibility results on adjuvant HAIP chemotherapy in patients with resectable CRLM. Some fundamental elements have been considered in the design of our program, including thorough training on all safety and technical aspects, selection of appropriate materials, and careful selection of patients and participating centers. The number of participating centers for the safety and feasibility study was only two, to guarantee adequate training and experience. All future implantations will be performed by a team of two experienced surgeons, and new surgeons will only be allowed to perform implantations after thorough training to sustain knowledge and skills.

After confirming safety and feasibility, we proceeded with a multicenter, phase III RCT (the PUMP trial) to study the effectiveness of adjuvant HAIP chemotherapy in patients with resectable CRLMs (www.trialregister.nl: NTR7493). ${ }^{20}$

This study showed that starting an HAIP chemotherapy program can be safe and feasible after adequate training and proctoring of a multidisciplinary team.

ACKNOWLEDGMENT None.

FUNDING Funding was received from Erasmus MC Efficiency (Mrace), Erasmus MC Foundation, Coolsingel Foundation, and Tricumed GmBh (in kind).

DISCLOSURE Florian E. Buisman, Dirk J. Grünhagen, Marjolein Y. V. Homs, Cecile Grootscholten, Wills F. Filipe, Nancy E. Kemeny, Andrea Cercek, Micheal I. D’Angelica, Maarten L. Donswijk, Leni van Doorn, Jasper Emmering, William R. Jarnagin, T. Peter Kingham, Elisabeth G. Klompenhouwer, Niels F. M. Kok, Maria C. Kuiper, Adriaan Moelker, Warner Prevoo, Michelle W. J. Versleijen, Cornelis Verhoef, Koert F. D. Kuhlmann, and Bas Groot Koerkamp have no conflicts of interest to declare.

OPEN ACCESS This article is distributed under the terms of the Creative Commons Attribution 4.0 International License (http://crea tivecommons.org/licenses/by/4.0/), which permits unrestricted use, distribution, and reproduction in any medium, provided you give appropriate credit to the original author(s) and the source, provide a link to the Creative Commons license, and indicate if changes were made.

\section{REFERENCES}

1. Kanas GP, Taylor A, Primrose JN, et al. Survival after liver resection in metastatic colorectal cancer: review and meta-analysis of prognostic factors. Clin Epidemiol. 2012;4:283-301.

2. Ackerman NB. The blood supply of experimental liver metastases. IV. Changes in vascularity with increasing tumor growth. Surgery. 1974;75(4):589-596. 
3. Breedis C, Young G. The blood supply of neoplasms in the liver. Am J Pathol. 1954;30(5):969-977.

4. Ensminger WD, Gyves JW. Clinical pharmacology of hepatic arterial chemotherapy. Semin Oncol. 1983;10(2):176-182.

5. Kemeny NE, Chou JF, Boucher TM, et al. Updated long-term survival for patients with metastatic colorectal cancer treated with liver resection followed by hepatic arterial infusion and systemic chemotherapy. J Surg Oncol. 2016;113(5):477-484.

6. Kemeny NE, Gonen M. Hepatic arterial infusion after liver resection. N Engl J Med. 2005;352(7):734-735.

7. Lorenz M, Muller HH, Schramm H, et al. Randomized trial of surgery versus surgery followed by adjuvant hepatic arterial infusion with 5-fluorouracil and folinic acid for liver metastases of colorectal cancer. German Cooperative on Liver Metastases (Arbeitsgruppe Lebermetastasen). Ann Surg. 1998;228(6):756-762.

8. Allen PJ, Nissan A, Picon AI, et al. Technical complications and durability of hepatic artery infusion pumps for unresectable colorectal liver metastases: an institutional experience of 544 consecutive cases. J Am Coll Surg. 2005;201(1):57-65.

9. Chakedis J, Beal EW, Sun S, et al. Implementation and early outcomes for a surgeon-directed hepatic arterial infusion pump program for colorectal liver metastases. $J$ Surg Oncol. 2018;118(7):1065-1073.

10. Kemeny MM, Goldberg D, Beatty JD, et al. Results of a prospective randomized trial of continuous regional chemotherapy and hepatic resection as treatment of hepatic metastases from colorectal primaries. Cancer. 1986;57(3):492-498.

11. Ammori JB, Kemeny NE. Regional hepatic chemotherapies in treatment of colorectal cancer metastases to the liver. Semin Oncol. 2010;37(2):139-148.

12. Power DG, Kemeny NE. The role of floxuridine in metastatic liver disease. Mol Cancer Ther. 2009;8(5):1015-1025.

13. Kemeny N, Huang Y, Cohen AM, et al. Hepatic arterial infusion of chemotherapy after resection of hepatic metastases from colorectal cancer. N Engl J Med. 1999;341(27):2039-2048.

14. Groot Koerkamp B, Sadot E, Kemeny NE, et al. Perioperative hepatic arterial infusion pump chemotherapy is associated with longer survival after resection of colorectal liver metastases: a propensity score analysis. J Clin Oncol. 2017;35(17):1938-1944.

15. Hamady ZZ, Lodge JP, Welsh FK, et al. One-millimeter cancerfree margin is curative for colorectal liver metastases: a propensity score case-match approach. Ann Surg. 2014;259(3):543-548.

16. Masi G, Loupakis F, Pollina L, et al. Long-term outcome of initially unresectable metastatic colorectal cancer patients treated with 5-fluorouracil/leucovorin, oxaliplatin, and irinotecan (FOLFOXIRI) followed by radical surgery of metastases. Ann Surg. 2009;249(3):420-425.

17. Seymour MT, Maughan TS, Ledermann JA, et al. Different strategies of sequential and combination chemotherapy for patients with poor prognosis advanced colorectal cancer (MRC FOCUS): a randomised controlled trial. Lancet. 2007;370(9582):143-152.

18. Nordlinger B, Sorbye H, Glimelius B, et al. Perioperative FOLFOX4 chemotherapy and surgery versus surgery alone for resectable liver metastases from colorectal cancer (EORTC 40983): long-term results of a randomised, controlled, phase 3 trial. Lancet Oncol. 2013;14(12):1208-1215.

19. Goéré D, Pignon JP, Gelli M, et al. Postoperative hepatic arterial chemotherapy in high-risk patients as adjuvant treatment after resection of colorectal liver metastases-a randomized phase II/ III trial-PACHA-01 (NCT02494973). BMC Cancer. 2018;18(1):787.

20. Buisman FE, Homs MYV, Grunhagen DJ, et al. Adjuvant hepatic arterial infusion pump chemotherapy and resection versus resection alone in patients with low-risk resectable colorectal liver metastases: the multicenter randomized controlled PUMP trial. BMC Cancer. 2019;19(1):327.

Publisher's Note Springer Nature remains neutral with regard to jurisdictional claims in published maps and institutional affiliations. 